\title{
Terapia oclusiva em ambliopia: fatores prognósticos
}

\author{
Occlusion therapy in amblyopia:factors that influence the outcome
}

\author{
Ana Carolina Fava Salata ${ }^{1}$ \\ Veridiana Toledo Nascimento Villaça ${ }^{2}$ \\ Renato Lopes Roma ${ }^{3}$ \\ Denise Yvonne Janovitz Norato ${ }^{4}$ \\ Keila Miriam Monteiro de Carvalho 5
}

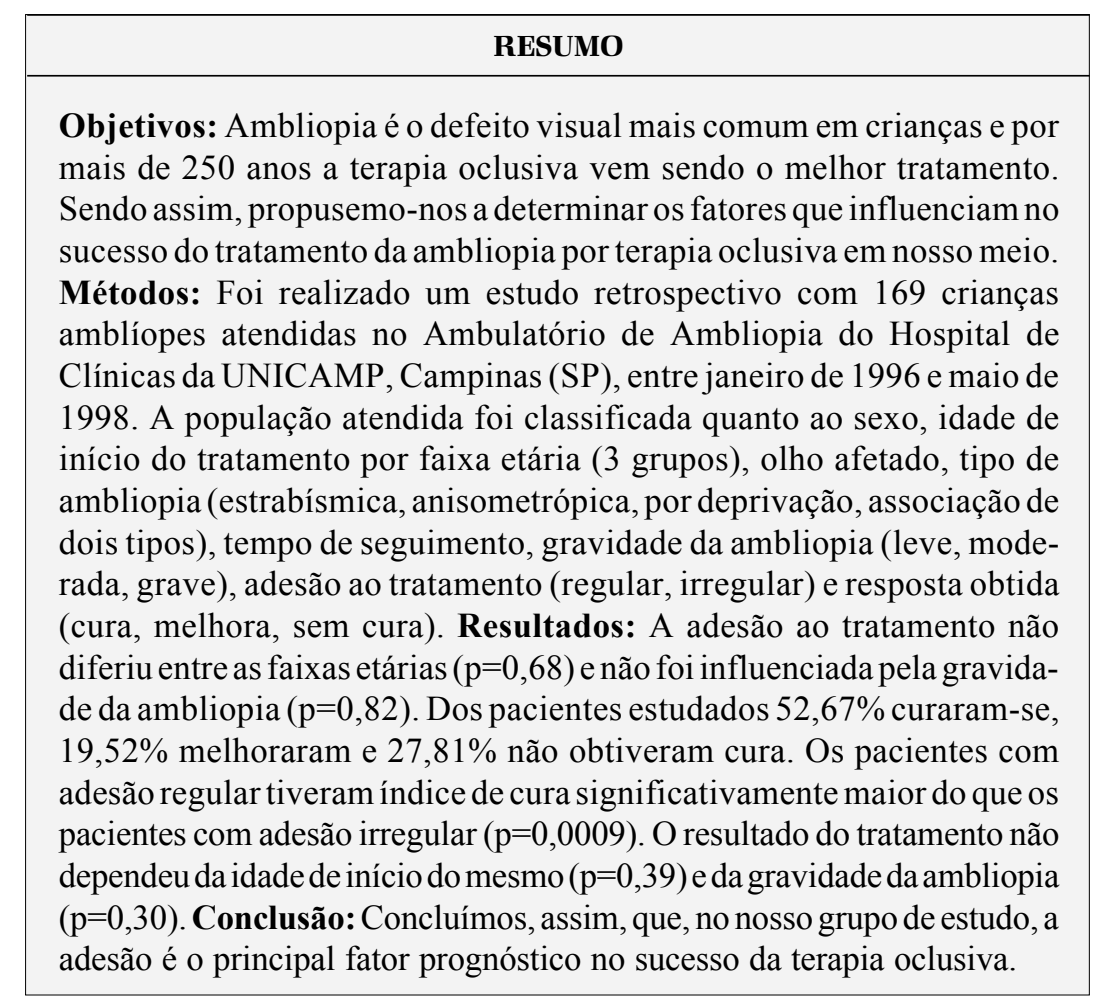

Descritores: Ambliopia/terapia; Prognósticos; Estrabismo; Curativos oclusivos; Cooperação do paciente
Apresentações em congressos:

XXX Congresso Brasileiro de Oftalmologia; Recife, 4 a 7 de Setembro de 1999.

$103^{\text {rd }}$ Annual Meeting of the American Academy of Ophthalmology, XXII Congress of the Pan-American Association of Ophthalmology; Orlando, Florida EUA, 24 a 27 de Outubro de 1999.

${ }^{1}$ Médica Residente

${ }^{2}$ Médica Voluntária

3 Acadêmico do sexto ano de graduação em Medicina

${ }^{4}$ Professor Assistente Doutor

${ }^{5}$ Professor Assistente Doutor

1,2,5 Departamento de Oftalmologia e Otorrinolaringologia da Faculdade de Ciências Médicas da Universidade Estadual de Campinas.

${ }^{4}$ Departamento de Genética Médica da Faculdade de Ciências Médicas da Universidade Estadual de Campinas.

Os autores declaram não possuir interesse financeiro no desenvolvimento do referido estudo.

Endereço para correspondência: Ana Carolina Fava Salata - Rua Conselheiro Paula Souza, 182 - Campinas (SP) CEP 13083-080.

E-mail:keila.carvalho@merconet.com.br

\section{INTRODUÇÃO}

A ambliopia é definida como baixa acuidade visual uni ou bilateral sem lesão orgânica aparente. Suas principais causas são estrabismo, anisometropia, altas ametropias não corrigidas precocemente e deprivação visual por falta de estímulo(1). É o defeito visual mais comum em crianças, com prevalência de 3 a $5 \%^{(2-3)}$, e seu diagnóstico e tratamento precoces trazem resultados satisfatórios ${ }^{(4)}$.

Há mais de duzentos e cinqüenta anos o tratamento mais utilizado para ambliopia consiste na oclusão do olho são para que haja estímulo do olho afetado $^{(5-6)}$, porém sua eficácia permanece incerta com relatos de sucesso variando de 30 a $92 \%{ }^{(7-8)}$. Apesar destes dados, a terapia oclusiva continua sendo o melhor tratamento ${ }^{(5,9)}$.

A penalização já foi utilizada como tratamento na tentativa de melhorar a adesão, porém foi abandonada por muitos autores por não oferecer resultados vantajosos em relação à oclusão e ainda poder causar prejuízo ao olho dominante ${ }^{(10)}$. 
A adesão ao tratamento por terapia oclusiva, largamente estudada e discutida, depende da idade do paciente no início do tratamento, da acuidade visual inicial com correção, do nível sócio-econômico do paciente e da compreensão dos pais ${ }^{(3,11)}$. A não adesão, contudo, chega a cifras de $50 \%^{(12)}$ e como fatores contribuintes são citadas a terapia prolongada e a não aceitação da criança ${ }^{(11)}$.

Sendo assim, o objetivo deste trabalho é avaliar a adesão ao tratamento da ambliopia por terapia oclusiva e a resposta obtida na população atendida em um hospital universitário, determinando os fatores que influenciam o sucesso do tratamento em nosso meio.

\section{MÉTODOS}

Foi realizado um estudo retrospectivo de 169 crianças amblíopes, acompanhadas no Ambulatório de Ambliopia do Hospital de Clínicas da Universidade Estadual de Campinas, no período de Janeiro de 1996 a Maio de 1998.

Todas as crianças foram submetidas a exame oftalmológico completo, que incluía medida da acuidade visual (cartões de Teller para crianças que não informavam a acuidade, tabela de Snellen para aquelas que informavam), teste de cobertura simples e alternado, teste de cobertura com prisma, quando havia acuidade visual suficiente para fixação do olho, caso contrário a medida do desvio foi feita pelo método de Krimsky, refração estática com ciclopentolato, refração subjetiva quando possível, estereoacuidade e oftalmoscopia direta e indireta.

A população atendida foi classificada quanto ao sexo, idade de início do tratamento, olho afetado, tipo de ambliopia, tempo de seguimento, gravidade da ambliopia, aderência ao tratamento e resposta obtida.

A idade foi analisada por faixa etária totalizando 3 grupos: grupo 1 - de 0 a 2 anos e onze meses de idade, com 80 crianças; grupo 2- de 3 a 5 anos e onze meses, com 69 e grupo 3- de 6 a 8 anos e onze meses de idade, com 20 crianças.

A ambliopia foi classificada conforme o proposto por von Noorden ${ }^{(1)}$ sendo o critério adotado: estrabísmica, refracional iso ou anisometrópica, por deprivação e associação de 2 tipos. A gravidade da ambliopia foi classificada em leve nos casos em que a acuidade visual corrigida no pior olho foi maior que 0,5 (45 casos); moderada quando entre 0,5 e 0,3 (49 casos) e grave se foi menor ou igual a 0,3 no pior olho ( 75 casos).

A adesão ao tratamento foi considerada regular quando a oclusão prescrita foi utilizada corretamente e o paciente compareceu a todas as consultas marcadas e irregular quando o paciente não compareceu às consultas marcadas e/ou não usou corretamente a oclusão.

A oclusão prescrita seguiu o padrão utilizado no serviço, que difere de acordo com a gravidade da ambliopia e a idade da criança. (na coluna a seguir)

A oclusão não deveria ser suspensa até a idade de oito anos e onze meses.

O sucesso do tratamento foi atingido quando a acuidade

\begin{tabular}{|llll|}
\hline \multirow{2}{*}{$\begin{array}{l}\text { 0 a } 2 \text { anos e } \\
\text { onze meses }\end{array}$} & $\begin{array}{l}\text { 3 a 5anos e } \\
\text { onze meses }\end{array}$ & $\begin{array}{l}\text { 6 a } 8 \text { anos e } \\
\text { onze meses }\end{array}$ \\
& A: $1 \times 1$ & A: $3 \times 1$ & A: $5 \times 1$ \\
Moderada & B: 2 horas/dia & B: 4 horas/dia & B: 6 horas/dia \\
& A: $2 \times 1$ & A: $4 \times 1$ & A: $6 \times 1$ \\
Grave & B: 3 horas/dia & B: 5 horas/dia & B: 8 horas/dia \\
& A: $3 \times 1$ & A: $5 \times 1$ & A: total 15 dias \\
Sendo: A: oclusão total para pacientes com desvio manifesto maior que 20 \\
dioptrias; B: oclusão parcial para portadores de anisometropia, desvio inter- \\
mitente, foria e desvio manifesto menor que 20 dioptrias
\end{tabular}

visual em cada olho diferiu no máximo uma linha, segundo tabela de Snellen (critério de cura). Nos casos em que o critério de cura não foi alcançado, os pacientes foram classificados como "melhora" se obtiveram pelo menos duas linhas de melhora na acuidade visual, e "sem cura" aqueles que não apresentaram qualquer melhora.

Para análise estatística dos dados foi utilizado o teste do Qui-quadrado $\left(\chi^{2}\right)$.

\section{RESULTADOS}

Foram analisadas 169 crianças amblíopes, sendo 76 $(44,97 \%)$ do sexo masculino e $93(55,03 \%)$ do sexo feminino. O tempo de seguimento variou de 0 a 8 anos, sendo que 126 pacientes $(75,15 \%)$ foram acompanhados de 2 meses a 2 anos.

Entre os pacientes estudados, 69 (40,82\%) tinham o olho direito afetado, 75 (44,38\%), o olho esquerdo e $25(14,80 \%)$ tinham ambos os olhos afetados.

A maioria das ambliopias era do tipo estrabísmica, num total de $127(75,15 \%)$ casos; $7(4,14 \%)$ eram do tipo anisometrópica; $21(12,42 \%)$, por deprivação e $14(8,29 \%)$ crianças tinham ambliopia de causas associadas.

Não houve diferença significativa na gravidade das ambliopias de acordo com a faixa etária de início do tratamento $\left(\chi^{2}=6,819 ; \mathrm{p}=0,15\right)$ (Figura 1$)$ e a idade de início do tratamento não influenciou significativamente o resultado (Tabela 1 ).

Entre todos os pacientes, $47(27,81 \%)$ não obtiveram cura; $33(19,52 \%)$ obtiveram melhora da acuidade visual e 89 $(52,67 \%)$ foram curados (Figura 2 ).

A adesão ao tratamento oclusivo não diferiu significativamente nas 3 faixas etárias, sendo de $46,25 \%$ de 0 a 2 anos e onze meses; $52,17 \%$ de 3 a 5 anos e onze meses e de $55,00 \%$ de 6 a 8 anos e onze meses (Tabela 2).

A adesão foi regular em $84(49,70 \%)$ dos pacientes e irregular em 85 (50,30\%). Dos pacientes com adesão regular, 55 $(65,47 \%)$ obtiveram cura, $12(14,28 \%)$ melhoraram e $17(20,23 \%)$ não obtiveram cura. Já, entre os pacientes com adesão irregular, $34(40,00 \%)$ foram curados, $21(24,70 \%)$ melhoraram e $30(35,30 \%)$ não obtiveram cura (Tabela 3 ). Os pacientes com adesão regular tiveram um índice de cura $(65,47 \%)$ significativamente maior dos que os pacientes com adesão irregular $(40,00 \%)\left(\chi^{2}=10,999\right.$; $\mathrm{p}=0,0009)$. Entre os pacientes que não obtiveram cura e aqueles que apenas melhoraram, não se detectou diferença significativa quanto à adesão ao tratamento $\left(\chi^{2}=0,000 ; \mathrm{p}=0,99\right)$. 


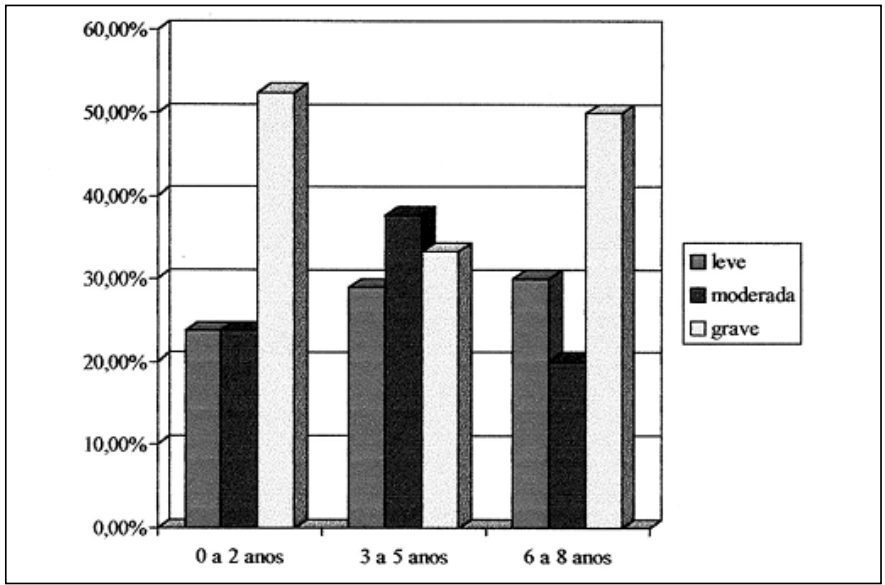

Figura 1 - Histograma da gravidade da ambliopia pela faixa etária

Tabela 1. Resultado do tratamento de acordo com faixa etária

\begin{tabular}{lcccc}
$\begin{array}{l}\text { Faixa etária/ } \\
\text { resultado }\end{array}$ & $\begin{array}{c}\text { Cura } \\
(\mathbf{n = 8 9})\end{array}$ & $\begin{array}{c}\text { Melhora } \\
(\mathbf{n}=\mathbf{3 3})\end{array}$ & $\begin{array}{c}\text { Sem cura } \\
(\mathbf{n = 4 7})\end{array}$ & $\begin{array}{c}\text { Total } \\
(\mathbf{n}=169)\end{array}$ \\
$\begin{array}{l}0 \text { a } 2 \text { anos e } \\
\text { onze meses }\end{array}$ & $39(48,75 \%)$ & $17(21,25 \%)$ & $24(30,00 \%)$ & 80 \\
$\begin{array}{l}3 \text { a } 5 \text { anos e } \\
\text { onze meses }\end{array}$ & $42(60,87 \%)$ & $12(17,39 \%)$ & $15(21,74 \%)$ & 69 \\
$\begin{array}{l}6 \text { a } 8 \text { anos e } \\
\text { onze meses }\end{array}$ & $8(40,00 \%)$ & $4(20,00 \%)$ & $8(40,00 \%)$ & 20 \\
$\chi^{2}=4,130 ; p=0,39$ & & & \\
\hline
\end{tabular}

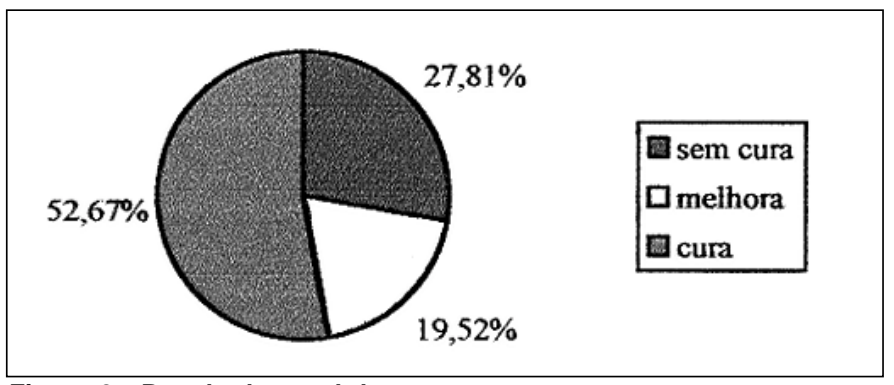

Figura 2 - Resultado geral do tratamento

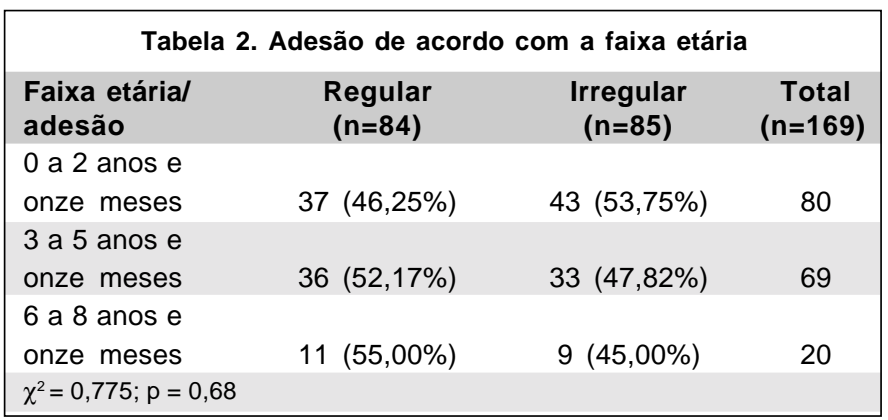

A gravidade da ambliopia não influenciou significativamente a adesão ao tratamento oclusivo (Tabela 4), tendo 35 (46,67\%) pacientes apresentado cura no grupo de ambliopia grave, 31 $(63,26 \%)$ no moderado e $23(51,11 \%)$ no leve (Tabela 5).
Tabela 3. Resultado do tratamento de acordo com adesão

\begin{tabular}{lcccc}
$\begin{array}{l}\text { Adesão/ } \\
\text { resultado }\end{array}$ & $\begin{array}{c}\text { Cura } \\
(\mathbf{n}=89)\end{array}$ & $\begin{array}{c}\text { Melhora } \\
(\mathbf{n}=33)\end{array}$ & $\begin{array}{c}\text { Sem cura } \\
(\mathbf{n}=\mathbf{4 7})\end{array}$ & $\begin{array}{c}\text { Total } \\
(\mathbf{n}=169)\end{array}$ \\
Regular & $55(65,47 \%)$ & $12(14,28 \%)$ & $17(20,23 \%)$ & 84 \\
Irregular & $34(40,00 \%)$ & $21(24,70 \%)$ & $30(35,30 \%)$ & 85 \\
$\chi^{2}=11,000 ; p=0,004$ & & & \\
\hline
\end{tabular}

Tabela 4. Adesão de acordo com gravidade da ambliopia

\begin{tabular}{lccc|}
$\begin{array}{l}\text { Gravidade/ } \\
\text { adesão }\end{array}$ & $\begin{array}{c}\text { Regular } \\
(\mathbf{n = 8 4 )}\end{array}$ & $\begin{array}{c}\text { Irregular } \\
(\mathbf{n = 8 5})\end{array}$ & $\begin{array}{c}\text { Total } \\
(\mathbf{n = 1 6 9 )}\end{array}$ \\
Leve & $21(25,00 \%)$ & $24(28,23 \%)$ & 45 \\
Moderada & $26(30,95 \%)$ & $23(27,05 \%)$ & 49 \\
Grave & $37(44,05 \%)$ & $38(44,72 \%)$ & 75 \\
$\chi^{2}=0,391 ; p=0,82$ & & & \\
\hline
\end{tabular}

Tabela 5. Resultado do tratamento de acordo com a gravidade

\begin{tabular}{lcccc}
$\begin{array}{l}\text { Resultado/ } \\
\text { gravidade }\end{array}$ & $\begin{array}{c}\text { Cura } \\
(\mathbf{n}=89)\end{array}$ & $\begin{array}{c}\text { Melhora } \\
(\mathbf{n}=33)\end{array}$ & $\begin{array}{c}\text { Sem cura } \\
(\mathbf{n}=47)\end{array}$ & $\begin{array}{c}\text { Total } \\
(\mathbf{n}=169)\end{array}$ \\
Leve & $23(51,11 \%)$ & $8(17,77 \%)$ & $14(31,12 \%)$ & 45 \\
Moderada & $31(63,26 \%)$ & $6(12,24 \%)$ & $12(24,49 \%)$ & 49 \\
Grave & $35(46,67 \%)$ & $19(25,33 \%)$ & $21(28,00 \%)$ & 75 \\
$\chi^{2}=4,646 ; p=0,30$ & & & \\
\hline
\end{tabular}

\section{DISCUSSÃO}

Muitos estudos mostram que os principais fatores prognósticos no tratamento da ambliopia por terapia oclusiva são a idade de início do tratamento, a gravidade da ambliopia e a adesão ao tratamento ${ }^{(3,12)}$.

No nosso grupo de estudo o resultado do tratamento não dependeu da idade de início do tratamento, concordando com os resultados de Beardsell et al. ${ }^{(13)} \mathrm{e}$ Woodruff et al..$^{(14)}$. O fato de terem sido obtidos bons índices de cura, mesmo com crianças que iniciaram o tratamento mais tarde, pode indicar que ainda haveria plasticidade neural para reverter a privação visual que ocorreu no corpo geniculado lateral e córtex visual ${ }^{(15)}$.

No presente trabalho a adesão não dependeu da faixa etária, diferindo de Nucci et al. ${ }^{(12)}$, que sugeriu que as crianças mais velhas teriam melhor adesão, decorrente da melhor compreensão do problema e colaboração com o tratamento.

Embora na literatura encontrem-se diversas referências relatando que quanto maior a privação visual, pior o resultado ${ }^{(4,13-14)}$, neste estudo não houve associação entre o resultado e a gravidade da ambliopia, o que poderia ser explicado pela inexistência no nosso grupo de uma associação entre a gravidade e a adesão; não tendo a adesão ao tratamento sido influenciada pela gravidade da ambliopia. Smith et al..$^{(3)}$ e Souza et al. ${ }^{(4)}$ também referiram não ter observado a associação entre adesão e gravidade, enquanto Nucci et al. ${ }^{(12)}$ encontraram que quanto maior a gravidade pior a adesão. 
Em $75,15 \%$ dos pacientes estudados o tempo de seguimento foi de 2 meses a 2 anos. É possível que existam casos de ambliopia estrabísmica que não se curaram, pois o tempo de seguimento foi curto.

Os pacientes com adesão regular ao tratamento tiveram índice de cura significativamente maior que aqueles com adesão irregular, como seria esperado, estando o nosso índice de cura $(65,47 \%)$ intermediário aos observados na literatura, que variaram entre 30 a $92 \%{ }^{(7-8)}$. Essa variação, observada nos diferentes trabalhos, pode ser decorrente do critério de cura adotado pelos autores. Não detectamos diferenças da adesão entre o grupo de pacientes que apresentaram apenas melhora da acuidade visual e os considerados não curados, mas isto pode ter ocorrido devido à dificuldade de mensuração da adesão ao tratamento, pois não foram separados os pacientes que, apesar da adesão irregular, usaram parcialmente a oclusão prescrita e os que não a usaram.

Concluímos, assim, que, no nosso grupo de estudos, a adesão ao tratamento é o principal fator prognóstico na cura da ambliopia.

\section{ABSTRACT}

Purpose: Amblyopia is the most common form of visual problem in children and for more than 250 years occlusion therapy is the standard treatment. Thus our purpose is to identify the factors that influence the outcome of amblyopia treatment with occlusion therapy. Methods: We reviewed 169 amblyopic children seen in the outpatient clinic of amblyopia of the Campinas State University, between January 1996 and May 1998. Patients were analyzed regarding sex, age at start of treatment (3 groups), affected eye, type of amblyopia (strabismic, anisometropic, visual deprivation, associated), follow-up, initial visual acuity (light, moderate, severe), compliance with treatment (good, poor) and outcome (fully treated, partially treated, not treated). Results: Compliance was not seen to be significantly related to age at start of treatment $(\mathrm{p}=0.68)$ or initial visual acuity $(\mathrm{p}=0.82) .52 .67 \%$ of the patients were fully treated while
$19.52 \%$ were partially treated and $27.81 \%$ were not treated. Children recorded as showing good compliance had a significantly better outcome than those with poor compliance $(p=0.0009)$. Neither the age at start of treatment $(p=0.39)$ nor the initial visual acuity $(\mathrm{p}=0.30)$ were significantly correlated with the final outcome. Conclusions: We concluded that the main factor affecting the final outcome of amblyopia treatment is compliance.

Keywords: Amblyopia/therapy; Prognosis; Strabismus; Occlusive dressings; Patient compliance

\section{REFER̂̂NCIAS}

1. Von Noorden GK. Binocular vision and ocular motility: theory and management of strabismus. $4^{\text {th }}$ ed. St. Louis, USA, C.V. Mosby; 1990. p. 208-45.

2. Kara-José N, Carvalho KM, Caldato R, Pereira V, Oliveira AM, Fonseca Neto JC. Atendimento de amblíopes e prevalência na população pré-escolar, Campinas, São Paulo, Brasil. Bol. Oficina Sanit Panam 1984;96:31-7.

3. Smith LK, Thompson JR, Woodruff G, Hiscox F. Factors affecting treatment compliance in amblyopia. J Pediatr Ophthalmol Strabismus 1995;32:98-101.

4. Souza EC, Carvalho AKR, Pulchinelli A, Gass C, Rothe E, Vanzella LL, et al. Cura e recorrência da ambliopia após terapia oclusiva. Arq Bras Oftalmol 1994;57:16-9.

5. Monteiro de Carvalho KM. Tratamiento de la ambliopía. In: Yllanes MEA, Prieto Diaz J, Souza Dias C, Restrepo GV. Actualidades del estrabismo latinoamericano. México: Consejo Latinoamericano de Estrabismo; 1998. p. 19-24.

6. Fielder AR, Auld R, Irwin M, Cocker KD, Jones HS, Moseley MJ. Compliance monitoring in amblyopia therapy. Lancet. 1994;343:547.

7. Hiscox F, Strong N, Thompson JR, Minshull C, Woodruff G. Occlusion for amblyopia: a comprehensive survey of outcome. Eye 1992;6:300-4.

8. Monteiro de Carvalho KM, Simões MST, Sanabria LBG, Kara-José N. Ambliopia: necessidade de prevenção pré-escolar. Arq Bras Oftalmol 1996; 59:354.

9. Campos EC. Update on strabismus and amblyopia. Acta Ophthalmol Scand 1995;214:17-24.

10. Gurovich L. Ambliopia. In: Prieto-Diaz J, Souza-Dias C. Estrabismo. Argentina: La Plata; 1996. p. 55-91.

11. Serpa G, Carvalho KMM, Kara-José N. Ambliopia: Aderência ao tratamento. Arq Bras Oftalmol 1995;58:226.

12. Nucci P, Alfarano R, Piantadina A, Brancato R. Compliance in antiamblyopia occlusion therapy. Acta Ophthalmol 1992;70:128-31.

13. Beardsell R, Clarke S, Hill M. Outcome of occlusion treatment for amblyopia. J Pediatr Ophthalmol Strabismus 1999;36:19-24.

14. Woodruff G, Hiscox F, Thompson JR, Smith LK. Factors affecting the outcome of children treated for amblyopia. Eye 1994;8:627-31.

15. Campos E. Amblyopia. Surv Ophthalmol 1995;40:23-39.

\title{
Novidades na Internet!!!
}

\author{
Agora no site CBO você tem disponível todas as informações na íntegra dos \\ Arquivos Brasileiros deOftalmologia \\ http://www.cbo.com.br/abo
}

